\title{
Pseudoaneurisma sobre válvula aórtica bicúspide simulando síndrome coronario agudo
}

\author{
Clares-Montón P. ${ }^{1 a}$, Martí-Sánchez D. ${ }^{2 a}$, de Agustín-Loeches JA. ${ }^{3}$, Sáenz-Casco LV. ${ }^{4}$, \\ Palazuelos-Molinero J.2a, Méndez-Fernández M. ${ }^{\text {5a }}$
}

Sanid. mil. 2014; 70 (4): 270-273; ISSN: 1887-8571

\begin{abstract}
RESUMEN
Presentamos el caso de varón joven que ingresa en Unidad Cuidados Intensivos con dolor torácico como manifestación de insuficiencia aórtica masiva secundaria a endocarditis infecciosa sobre válvula aórtica bicúspide. El ecocardiograma transesofágico muestra además como complicación grave pseudoaneurisma en seno de Valsalva que comunica tanto con raíz aórtica como con ventrículo izquierdo.
\end{abstract}

PALABRAS CLAVE: Endocarditis infecciosa, Válvula aórtica nativa, Pseudoaneurisma del seno de Valsalva, Insuficiencia aórtica severa, Síndrome aórtico agudo.

\section{Pseudoaneurysm of bicuspid aortic valve simulating acute coronary syndrome}

SUMMARY: We present the case of a young man who is admitted in Intensive Care Unit with chest pain as manifestation of masive aortic regurgitation due to infective endocarditis in bicuspid aortic valve. The transesophageal echocardiography also shows, as severe complication, pseudoaneurysm of the sinus of Valsalva that communicates with both aortic root as left ventricle.

KEY WORDS: Infective endocarditis, Aortic native valve, Sinus of Valsalva pseudoaneurysm, Severe aortic regurgitation, Aortic acute syndrome.

\section{INTRODUCCIÓN}

La causa más frecuente de insuficiencia aórtica crónica es la fiebre reumática. Sin embargo en el caso de afectación aguda las etiologías responsables más habituales son la endocarditis infecciosa por afectación valvular y la patología aórtica por dilatación del anillo.

Las manifestaciones clínicas de las endocarditis más frecuentes son, además de la afectación del estado general, la presencia de soplo cardíaco, fiebre o fenómenos embólicos ${ }^{1}$. Otras menos frecuentes son la presencia de arritmias, pericarditis o insuficiencia cardíaca aguda generalmente asociada a insuficiencia valvular como ocurre en el caso que presentamos a continuación.

\section{DESCRIPCIÓN CASO}

Se trata de un varón de 36 años fumador, bebedor de 52 gramos diarios de alcohol, consumidor ocasional de cocaína, sin historia cardiovascular conocida que acude a Urgencias por

${ }^{1}$ MIR

${ }^{2}$ Médico adjunto.

${ }^{3}$ Médico adjunto. Hospital Clínico San Carlos. Servicio de Cardiología.

${ }^{4}$ Cte. Médico. Hospital Central de la Defensa Gómez Ulla. Servicio de Medicina Intensiva.

${ }^{5}$ Cor. Médico. Hospital Central de la Defensa Gómez Ulla. Servicio de Cardiología. Madrid. España.

Dirección para correspondencia: patri_clares@hotmail.com.

Recibido: 29 de noviembre de 2013

Aceptado: 17 de marzo de 2014 presentar en reposo, dolor centrotorácico opresivo, transfixivo hacia la espalda, acompañado de intensa disnea y cortejo vegetativo. Refiere, cuatro días antes del episodio, escalofríos sin fiebre termometrada y tos seca con expectoración leve, por la cual recibió tratamiento con amoxicilina y broncodilatadores, así como astenia intensa y disnea de moderados esfuerzos desde hace un año.

En urgencias, impresiona de mal estado general, afebril, taquicárdico, con soplo diastólico III/IV panfocal, de predominio en foco aórtico, con TA de 95/68mmHg y crepitantes bibasales.

En electrocardiograma se objetiva taquicardia sinusal a 110 lpm con rectificación del segmento ST de V4 a V6. Los marcadores de daño miocárdico son negativos. La radiografía de tórax es normal. En analítica $\mathrm{Hb} 10,3$; plaquetas 142000; resto sin alteraciones. Ante la sospecha de síndrome aórtico agudo se realiza ecocardiograma transtorácico en el que se observa insuficiencia aórtica masiva (Figura 1) y tricuspídea III/IV con hipertensión pulmonar moderada, ventrículo izquierdo dilatado con hipoquinesia septal y función global $45 \%$ y TAC torácico que descarta patología aórtica aguda. Ingresa en unidad de cuidados intensivos donde permanece estable hemodinámicamente sin precisar soporte vasoactivo. Se realiza ecocardiograma transesofágico que confirma insuficiencia aórtica masiva sobre válvula aórtica bicúspide (Figura 2 y 3) con engrosamiento y fibrosis de la porción más anterior de los dos velos. Sobre el velo anterior presenta pseudoaneurisma de 2 × 2,5 cm que se comunica tanto con raíz aórtica como con cavidad ventricular izquierda ${ }^{2,3}$. Diámetro de aorta ascendente $38 \mathrm{~mm}$. Inversión holodiastólica del flujo en aorta torácica descendente. Función ventricular izquierda ligeramente deprimida. 


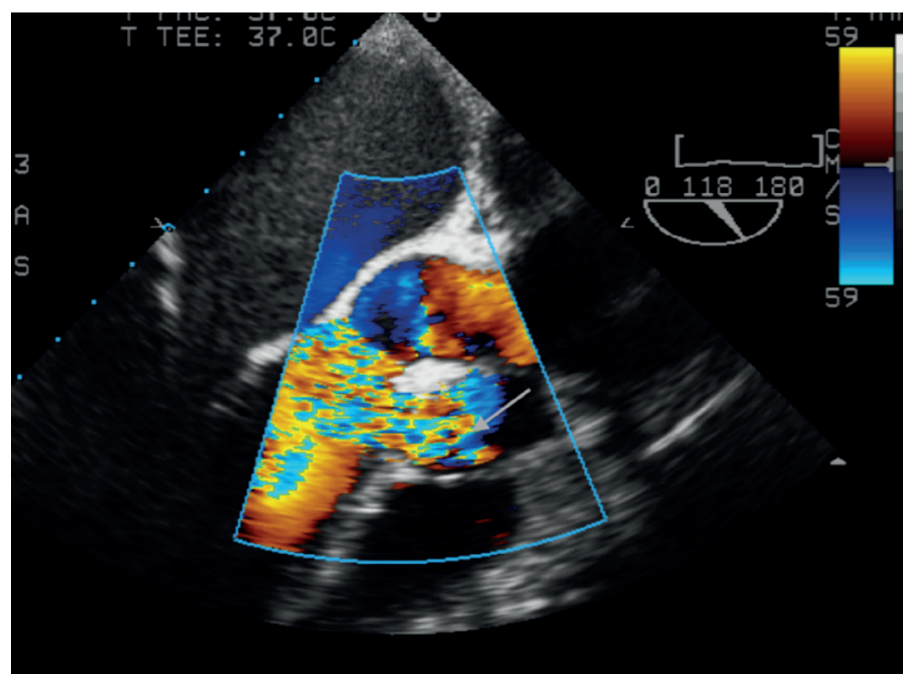

Figura 1. Ecocardiograma transesofágico que muestra insuficiencia aórtica masiva y pseudoaneurisma (flecha).

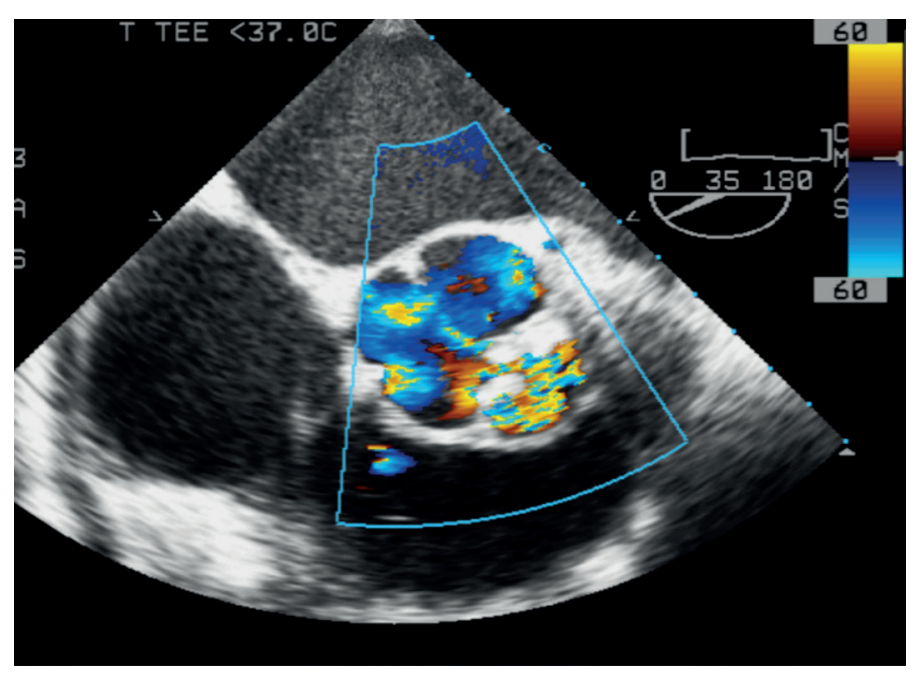

Figura 3. Ecocardiograma transesofágico. El Doppler color pone de manifiesto chorro de regurgitación sobre imagen de pseudoaneurisma.

Ante el diagnóstico de posible endocarditis con insuficiencia aórtica masiva se extraen hemocultivos y se inicia tratamiento antibiótico empírico con daptomicina y gentamicina.

Se realizó coronariografía sin encontrar lesiones angiográficamente significativas y en la aortografía se observa insuficiencia aórtica IV/IV muy excéntrica (Figura 4) con valva desestructurada que rellena retrógradamente ventrículo izquierdo. Sin datos de disección aórtica.

Debido a los hallazgos se realiza intervención quirúrgica urgente en la que se observa insuficiencia aórtica severa sobre válvula bicúspide desestructurada y calcificada con pseudoaneuris$\mathrm{ma}^{4-6}$ parcialmente roto a nivel seno coronariano derecho donde además se objetiva la presencia de un pseudoabceso. Se realiza sustitución valvular aórtica por prótesis metálica y reparación con parche de pericardio de pseudoabsceso en seno coronariano derecho. El paciente es extubado a las 4 horas de la cirugía y debido a postoperatorio sin complicaciones es trasladado a planta

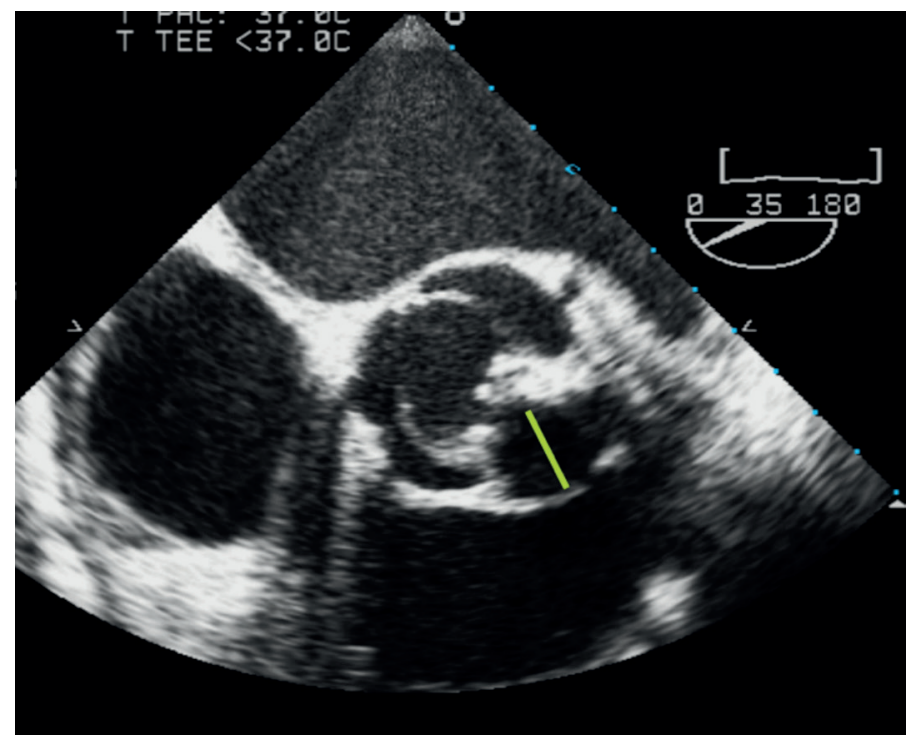

Figura 2. Ecocardiograma transesofágico. Apertura de válvula aórtica bicúspide con velos engrosados y ligeramente calcificados con pseudoaneurisma (linea verde).

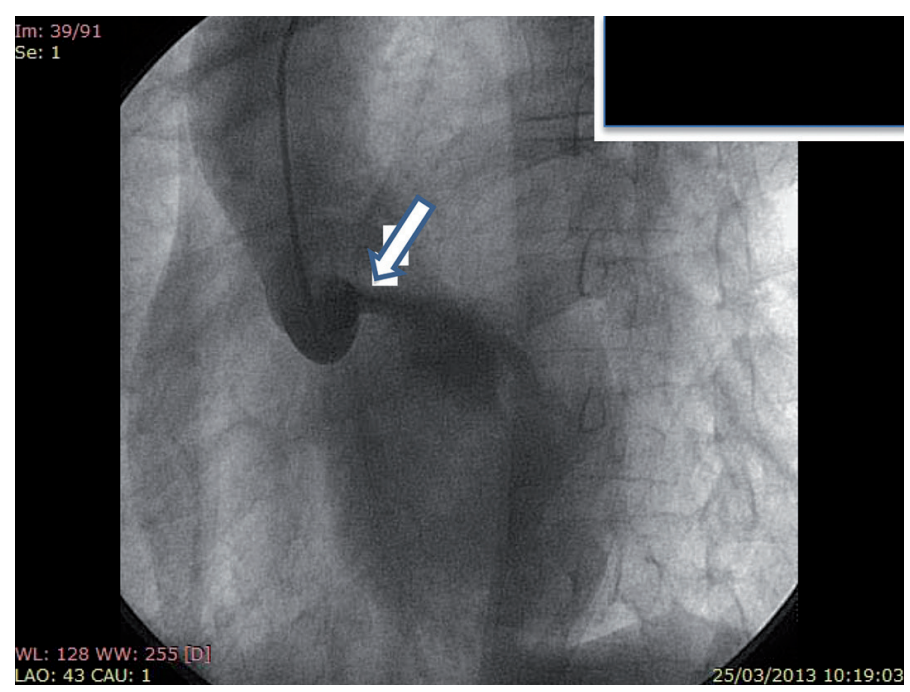

Figura 4. Aortografía que muestra insuficiencia aórtica masiva muy excéntrica.

donde se inicia tratamiento con IECAS y betabloqueantes tras observar disfunción sistólica severa en ecocardiograma de control.

Debido a la buena evolución y la sospecha de que se trate de una endocarditis subaguda en paciente con insuficiencia aórtica no conocida previamente sobre válvula bicúspide, y probablemente por patógeno derivado de la boca tras observar abundantes caries que el paciente no ha tratado por problemas económicos, se cambia daptomicina por ceftriaxona manteniéndose la gentamicina. Tanto hemocultivos como cultivo de la válvula para patógenos habituales y microorganismos tipo HACEK, Chlamydia, Coxiella y Brucella han resultado negativos En anatomía patológica de la válvula se visualiza inflamación aparentemente subaguda con macrófagos con hemosiderina, neovasos y calcificación valvular. 
El paciente fue dado de alta a domicilio tras 10 días de ingreso. En revisiones posteriores no se ha detectado ninguna incidencia y las serologías pendientes han resultado negativas.

\section{DISCUSIÓN}

La incidencia de endocarditis infecciosa (EI) es de 3,6 a 7 casos por 100.000 habitantes al año. En adultos jóvenes las causas predisponentes más frecuentes sobre válvula nativa son la enfermedad reumática, el prolapso valvular mitral, las enfermedades congénitas (como el ductus arterioso persistente, la comunicación interventricular o válvula aórtica bicúspide) o la adicción a drogas por vía parenteral. Los microorganismos implicados más frecuentes son los Estreptococos, S. aureus y Enterococos y en hasta un 10- 30\% de los pacientes el cultivo resulta negativo ${ }^{7,8}$.

El diagnóstico de la EI se establece según los criterios de Duke modificados, los cuales se basan en la presencia de hallazgos clínicos (soplo cardíaco de reciente aparición, fenómenos embólicos de origen desconocido, sepsis, fiebre asociada a otras circunstancias como presencia de material protésico cardíaco, historia previa de EI, valvulopatía o enfermedad congénita previa...), hallazgos ecocardiográficos y microbiológicos. En función de dichos hallazgos se clasifica la EI como definitiva o posible.

Actualmente la ecocardiografía transtorácica (ETT) y transesofágica (ETE) son fundamentales en el diagnóstico y posterior seguimiento de la EI. Los hallazgos considerados criterios mayores para el diagnóstico son la presencia de vegetación, absceso o nueva dehiscencia sobre válvula protésica. La sensibilidad del ETT varía de 40-63\% y de 90-100\% en ETE. En determinadas situaciones como la EI sobre dispositivos intracardíacos, valvulopatías severas previas, vegetaciones menores a $2 \mathrm{~mm}$ o EI sin vegetaciones el diagnóstico puede dificultarse particularmente. En algunos de estos casos la utilización de otras técnicas como la tomografía computerizada (TC), resonancia magnética (RM) o tomografía por emisión de positrones (PET), pueden ayudar en el diagnóstico.

$\mathrm{Su}$ tratamiento se basa en antibioterapia empírica inicialmente y dirigida tras conocer el microorganismo implicado y, en aproximadamente la mitad de los casos, existe también indicación para la realización de tratamiento quirúrgico. La cirugía debe considerar el recambio valvular en determinadas situaciones como en este caso son la insuficiencia cardíaca congestiva secundaria a disfunción valvular aórtica y la reparación de otras complicaciones intracardíacas como la invasión perivalvular con formación de abscesos y pseudo aneurismas cuya frecuencia es de 10-14 \% sobre válvulas nativas. En este caso se optó por la sustitución valvular aórtica por prótesis metálica, desbridamiento de la infección, extirpación y reconstrucción del daño anatómico con parche de pericardio.

A pesar de que la complicación más frecuente y grave de la EI es la aparición de insuficiencia cardíaca también debe considerarse la intervención quirúrgica precoz en caso de infección incontrolada o prevención de fenómenos embólicos. La infección incontrolada suele presentarse con persistencia de fiebre o hemocultivos positivos tras 7-10 días de tratamiento antibiótico correcto siempre que se hayan excluido otras causas. En estos casos, generalmente la causa de esta situación es la presencia de complicaciones como pseudoaneurismas, abscesos o fístulas por extensión de la infección al tejido perivalvular ${ }^{9}$. Estos fenómenos son más frecuentes en endocarditis sobre válvula aórtica que mitral ${ }^{10}$. La presencia de pseudoaneurismas y fístulas suele asociarse a importante daño sobre tejido valvular y perivalvular ${ }^{11,12}$. La técnica de elección para su diagnóstico y posterior seguimiento es la ecocardiografía transesofágica (ETE), puesto que la sensibilidad del ecocardiograma transtorácico es menor del $50 \%$. En caso de que los abscesos sean pequeños, especialmente si su localización es mitral y existe calcificación del anillo la ETE puede no visualizarlos. Otras complicaciones secundarias a la extensión de la infección son la presencia de defectos en tabique interventricular, afectación del sistema de conducción en forma de bloqueos auriculoventriculares y síndromes coronarios agudos. La endocarditis producida por microorganismos menos frecuentes, como los hongos ${ }^{13}$ o secundaria a organismos multirresistentes también debe ser tratada quirúrgicamente.

A pesar de que el riesgo embólico de la EI es muy elevado (20-50\%), el tratamiento antibiótico reduce este porcentaje a $6-20 \%{ }^{14}$. Sin embargo y debido a que el mayor riesgo embólico acontece en las primeras semanas de evolución, muchos pacientes ya han sufrido estos eventos en el momento del diagnóstico. Las localizaciones más frecuentes de estos fenómenos son el cerebro y bazo en EI izquierdas y pulmón en EI derechas y en pacientes portadores de marcapasos. Muchos son los factores que aumentan la probabilidad de embolismo: tamaño y movilidad de las vegetaciones, localización mitral de la EI, antecedente de embolismo previo, determinados microorganismos o afectación multivalvular de la EI. De todos ellos el tamaño y la movilidad de las vegetaciones son los predictores independientes más potentes de nuevos fenómenos embólicos ${ }^{15}$. El riesgo es mayor en las dos primeras semanas desde el inicio de tratamiento antibiótico y disminuye notablemente tras este período. La indicación de tratamiento quirúrgico debe individualizarse en cada caso sin olvidar que vegetaciones mayores de $15 \mathrm{~mm}$ son consideradas de muy alto riesgo embólico.

La morbimortalidad de la cirugía en la EI varía en función del agente infeccioso, la extensión a tejidos adyacentes, función ventricular izquierda y situación hemodinámica en el momento quirúrgico. Oscila entre el 5 y el 15\%16,17.

Las complicaciones postoperatorias son frecuentes. Entre ellas se encuentran la coagulopatía, resangrado de la herida quirúrgica, taponamiento cardíaco, insuficiencia renal aguda, ictus o bloqueos auriculoventriculares que precisen colocación de marcapasos.

La EI puede presentar otras complicaciones extracardíacas como la presencia de absceso esplénico, aneurismas micóticos, miocarditis, pericarditis y artritis séptica. Las complicaciones neurológicas son frecuentes $(20-40 \%)$ y generalmente consecuencia de fenómenos embólicos originados en las vegetaciones. El espectro clínico es muy amplio y varía desde ictus isquémicos o hemorrágicos, accidentes isquémicos transitorios, embolismos cerebrales silentes, aneurismas infecciosos, abscesos cerebrales, meningitis, encefalopatía tóxica hasta convulsiones. Todos aumentan la mortalidad de la EI, particularmente la aparición de ictus $^{18}$. El diagnóstico y tratamiento precoz es preciso para evitar su aparición o recurrencia. 


\section{Pseudoaneurisma sobre válvula aórtica bicúspide simulando síndrome coronario agudo}

\section{CONCLUSIONES}

La EI es una entidad relativamente frecuente que debe ser sospechada en pacientes con factores predisponentes o ante la presencia de hallazgos clínicos como los descritos. Requiere un diagnóstico y tratamiento precoz pues presenta una tasa de complicaciones y una morbimortalidad muy elevada. Dentro del diagnóstico el ecocardiograma transesofágico presenta un papel crucial tanto a la hora de establecer un juicio clínico como en el seguimiento y diagnóstico de complicaciones secundarias a la extensión de la infección sobre el tejido adyacente tan severas como la del caso que nos ocupa. La base del tratamiento es la antibioterapia a la que se une la cirugía en aproximadamente la mitad de los casos.

\section{BIBLIOGRAFÍA}

1. Htwe TH, Khardori NM. Cardiac emergencies: infective endocarditis, pericarditis, and miocarditis. Med Clin North Am 2012;96(6):1149-69.

2. Jang JJ, Battaglia KS, Kwai Ben VC. Sinus of valsalva pseudoaneurysm and mechanical aortic valve endocarditis. J Invasive Cardiol 2010;22(4):195.

3. Kanemitsu S, Tanabe S, Ohue K, Miyagawa H, Miyake Y, Okabe M. Aortic valve destruction and pseudoaneurysm of the sinus of Valsalva associated with infective endocarditis. Ann Thorac Cardiovasc Surg 2010;16(2):142-4.

4. Azevedo O, Ferreira F, Guardado J, Durães C, Quelhas I, Pereira A, et al. Mitral and aortic valve aneurysms secondary to infective endocarditis: impressive images of a rare echocardiographic finding. Eur $\mathrm{J}$ Echocardiogr. 2010;11(7):E28.

5. Tünel HA, Gülcan O, Demirtürk OS. Large pseudoaneurysm of the sinus of Valsalva after surgery for aortic valve endocarditis. J Heart Valve Dis. 2013 Mar: 22(2): 257-8.

6. Hori D, Noguchi K, Nomura Y, Tanaka H. Perivalvular pseudoaneurysm caused by streptococcus dysgalactiae in the presence of prosthetic aortic valve endocarditis. Ann Thorac Cardiovasc Surg. 2012; 18(3): 262-5.

7. Karchmer A W. Infective endocarditis. En: Bonow RO, Mann DL, Zipes DP, Libby P, editors. Braunwald's Heart Disease - A Textbook of Cardiovascular Medicine, Vol 1, 9th ed. Elsevier; 2011. p. 1540-1558.

8. Lamas CC, Eykyn SJ. Blood culture negative endocarditis: analysis of 63 cases presenting over 25 years. Heart 2003;89:258 - 262.
9. Anguera I, Miro JM, Evangelista A, Cabell CH, San Roman JA, Vilacosta I, Almirante B, Ripoll T, Farinas MC, Anguita M, Navas E, Gonzalez-Juanatey C, Garcia-Bolao I, Munoz P, de Alarcon A, Sarria C, Rufi G, Miralles F, Pare C, Fowler VG Jr, Mestres CA, de Lazzari E, Guma JR, Moreno A, Corey GR. Periannular complications in infective endocarditis involving native aortic valves. Am J Cardiol 2006;98:1254-1260.

10. Graupner C, Vilacosta I, SanRoman J, Ronderos R, Sarria C, Fernandez C, Mujica R, Sanz O, Sanmartin JV, Pinto AG. Periannular extension of infective endocarditis. J Am Coll Cardiol 2002;39:1204 - 1211.

11. Tingleff J, Egeblad H, Gotzsche CO, Baandrup U, Kristensen BO, Pilegaard $\mathrm{H}$, Pettersson G. Perivalvular cavities in endocarditis: abscesses versus pseudoaneurysms? A transesophageal Doppler echocardiographic study in 118 patients with endocarditis. Am Heart J 1995;130:93-100.

12. Anguera I, Miro JM, Vilacosta I, Almirante B, Anguita M, Munoz P, Roman JA, de Alarcon A, Ripoll T, Navas E, Gonzalez-Juanatey C, Cabell CH, Sarria C, Garcia-Bolao I, Farinas MC, Leta R, Rufi G, Miralles F, Pare C, Evangelista A, Fowler VG Jr, Mestres CA, de Lazzari E, Guma JR. Aorto-cavitary fistulous tract formation in infective endocarditis: clinical and echocardiographic features of 76 cases and risk factors for mortality. Eur Heart J 2005;26:288-297.

13. Garzoni C, Nobre VA, Garbino J. Candida parapsilosis endocarditis: a comparative review of the literature. Eur J Clin Microbiol Infect Dis 2007;26:915926.

14. Vilacosta I, Graupner C, San Roman JA, Sarria C, Ronderos R, Fernandez C, Mancini L, Sanz O, Sanmartin JV, Stoermann W. Risk of embolization after institution of antibiotic therapy for infective endocarditis. J Am Coll Cardiol 2002;39: 1489 - 1495.

15. Di Salvo G, Habib G, Pergola V, Avierinos JF, Philip E, Casalta JP, Vailloud JM, Derumeaux G, Gouvernet J, Ambrosi P, Lambert M, Ferracci A, Raoult D, Luccioni R. Echocardiography predicts embolic events in infective endocarditis. J Am Coll Cardiol 2001;37:1069-1076.

16. Delay D, Pellerin M, Carrier M, Marchand R, Auger P, Perrault LP, Hebert Y, Cartier R, Page P, Pelletier LC. Immediate and long-term results of valve replacement for native and prosthetic valve endocarditis. Ann Thorac Surg 2000;70: 1219 - 1223 .

17. David TE, Gavra G, Feindel CM, Regesta T, Armstrong S, Maganti MD. Surgical treatment of active infective endocarditis: a continued challenge. J Thorac Cardiovasc Surg 2007;133:144 - 149.

18. Thuny F, Avierinos JF, Tribouilloy C, Giorgi R, Casalta JP, Milandre L, Brahim A, Nadji G, Riberi A, Collart F, Renard S, Raoult D, Habib G. Impact of cerebrovascular complications on mortality and neurologic outcome during infective endocarditis: a prospective multicentre study. Eur Heart J 2007;28:1155-1161. 